INVESTIGACIÓN

https://doi.org/10.15198/seeci.2020.52.17-28

Recibido: 07/08/2019 --- Aceptado: 12/09/2019 --- Publicado: 15/07/2020

\title{
EDMODO: UNA PLATAFORMA DE E-LEARNING PARA LA INCLUSIÓN
}

\section{EDMODO: AN E-LEARNING PLATFORM FOR INCLUSION}

María Elena del Valle Mejías. Universidad Metropolitana de Caracas. Venezuela.

mvillalba@unimet.edu.ve

\section{RESUMEN}

El presente artículo resume la experiencia pedagógica de incluir dentro de los recursos educativos en un colegio y una universidad venezolana el LMS: edmodo. En el desarrollo del mismo se abordan: las características más importantes del entorno, las implicaciones de incluir en la enseñanza elementos tecnológicos y filosóficos provenientes del e-learning, la descripción del contexto socio-económico de Venezuela, el uso del LMS y los beneficios generados del mismo y finalmente las conclusiones a las que se llega luego de valorar el impacto de su aplicación. La plataforma de e-learning edmodo se ha constituido en un mecanismo de inclusión al ofrecer entornos de acceso gratuito, repositorios de recursos digitales y mecanismos de comunicación eficaces entre alumnos y profesores. Esto es parte lógica del proceso de adaptación a las nuevas tecnologías de la información por parte del sector educativo, siendo un indicativo de que la misma va mucho más allá de la arbitraria y cara distribución de equipos informáticos en las aulas: el camino lógico de esta adaptación, ante la experiencia general con las TIC, es el de crear herramientas de software que permitan el uso del hardware con fines específicamente académicos que, además, supongan algún tipo de ventaja o mejora con respecto a la clase analógica.

PALABRAS CLAVE: edmodo - inclusión - e-learning - Venezuela - TIC - software - plataformas.

\section{ABSTRACT}

This article summarizes the pedagogical experience of including the LMS: edmodo within the educational resources of a Venezuelan college and a university. In its

\footnotetext{
${ }^{1}$ María Elena del Valle Mejías: Doctora en Ciencias de la Educación en el Pedagógico de Caracas, postdoctorado en Semiótica y Pragmática en la Universidad Complutense de Madrid. Investigadora certificada de la Universidad Complutense de Madrid, el grupo internacional CONCILIUM y del CELARG. Directora del Master en Comunicación de la TECH University. Profesora Invitada de la Universidad de Vigo. Profesora Honorífica de Universidad Rey Juan Carlos. Colaboradora habitual de los Master en la ESERP y EAE.

mvillalba@unimet.edu.ve
} 
development, the most important characteristics of the environment, the implications of including, in teaching, the description of the socio-economic context of Venezuela, the use of the LMS and the benefits generated therefrom are addressed and, finally, the conclusions reached after assessing the impact of its application. The edmodo elearning platform has become an inclusion mechanism by offering free-access environments, repositories of digital resources and effective communication mechanisms between students and teachers. This is a logical part of the process of adaptation to the new information technologies by the educational sector, being an indication that it goes well beyond the arbitrary and expensive distribution of computer equipment in the classroom: the logical path of this adaptation, given the general experience with ICT, is to create software tools that allow us to use hardware for specifically academic purposes that, in addition, involve some type of advantage or improvement over the analogical class.

KEYWORDS: edmodo - inclusion - e-learning - Venezuela - ICT - software, platforms.

\section{EDMODO: UMA PLATAFORMA DE E-LEARNING PARA À INCLUSÃO}

\section{RESUMO}

O presente artigo resume a experiência pedagógica de incluir dentro dos recursos educativos de uma escola e uma universidade venezuelana o sistema de gestão de aprendizado: edmodo. No seu desenvolvimento são abordadas: as características mais importantes do entorno, as implicações de incluir no ensino, elementos tecnológicos e filosóficos que provém do e-learning, a descrição do contexto sócioeconômico da Venezuela, o uso do sistema de gestão de aprendizado e os benefícios gerados por ele, e finalmente as conclusões que são alcançadas após valorizar o impacto da sua aplicação. A plataforma de e-learning edmodo tem se constituído como um mecanismo de inclusão ao oferecer ambientes de acesso gratuito, repositórios de conteúdos digitais e mecanismos de comunicação eficazes entre estudantes e professores. Isto é parte da lógica do processo de adaptação às novas tecnologias de informação pelo setor educacional, sendo um indicativo de que a mesma vai além da arbitrária e cara de distribuição de equipamentos informáticos nas aulas: o caminho lógico desta adaptação, perante a experiência geral com as TIC, é criar ferramentas de software que permitam o uso de hardware com fins especificamente acadêmicos que, além disso, suponham algum tipo de vantagem ou melhoria com respeito as aulas analogicas.

PALAVRAS CHAVE: edmodo, inclusão, e-learning, Venezuela, TIC, software, plataformas. 


\section{Cómo citar el artículo:}

Del Valle Mejías, E. (2020). Edmodo: una plataforma de e-learning para la inclusión. [Edmodo: an e-learning platform for inclusion]. Revista de Comunicación de la SEECI, (52), 17-28. doi: http://doi.org/10.15198/seeci.2020.52.17-28

Recuperado de http://www.seeci.net/revista/index.php/seeci/article/view/613

\section{INTRODUCCIÓN}

Desde el inicio del siglo XXI, la educación en el mundo ha ido concretando cambios estructurales de importante valía. Sin duda alguna educar en este entorno implica hoy, múltiples habilidades y experticias. Nuestro interlocutor en el aula es, en muchos de los casos más diestro que su propio maestro en las herramientas tecnológicas. Pero el tener estas habilidades no garantiza en lo absoluto que posea el criterio formado para hacer uso racional y crítico de ellas.

Las plataformas de aprendizaje en la web se han ido multiplicando a medida que los años pasan, unas con mayor o menor tráfico pero sin duda alguna, muchas de ellas son el apoyo casi obligado de los maestros de hoy, como se desprende de Vaquerizo-García (2011) y de los apuntes sobre "mobile learning" de Alises Camacho (2017).

La estructura a desarrollar será la que sigue:

2. Edmodo: un LMS y una red social.

3. Venezuela, donde sobrevivir es la norma.

4. Beneficios de la plataforma en el contexto venezolano.

5 . Conclusiones y recomendaciones.

6. Bibliografía.

\section{EDMODO: UN LMS Y UNA RED SOCIAL}

Lo primero que haremos en este apartado es detenernos en las principales características de la plataforma que además la distinguen de las otras existentes. Describiremos los atributos de edmodo como entorno de aprendizaje colaborativo y profundizaremos en su potencial.

¿Cuáles son las características que debe tener todo LMS?

Según Clarenc (2012 y 2013) todas las plataformas de e-learning deberían cumplir con las siguientes:

\section{Interactividad}

Bedoya (2007) la definía como "la capacidad del receptor para controlar un mensaje no-lineal hasta el grado establecido por el emisor, dentro de los límites del medio de comunicación asincrónico". Los diferentes LMS, deben suministrar a través de cada uno de los recursos que proporcionan la posibilidad de que el alumno se relaciones en sus propios términos, con el entorno y con los materiales alojados en él. 


\section{Flexibilidad}

El LMS debe tener la propiedad de adaptarse a las necesidades del usuario así como también a sus posibilidades. En términos de tiempo y espacio, ofrecer la posibilidad al usuario de administrar de manera efectiva el tiempo del que dispone, así como también entrar y salir de la plataforma en los espacios acondicionados para ese propósito. Del mismo modo, la curva de aprendizaje, dentro del entorno fluctúa de acuerdo a las capacidades de cada usuario y respondiendo a los propósitos planteados por el arquitecto curricular.

\section{Escalabilidad}

Entendida como la capacidad operativa del entorno para no ver afectada su operatividad, por el número de usuarios conectados en simultáneo.

\section{Estandarización}

En este caso se refiere a un modelo común, compartido en alguna medida por los entornos de aprendizaje. (SCORM). Para cumplir con los objetivos planteados estos espacios cumplen con unas normas explícitas o implícitas a través de las cuáles se garantiza el logro de un modelo estándar que acopla los elementos que están presentados de manera independiente.

\section{Ubicuidad}

Vinculada con la omnipresencia, la ubicuidad se define como la capacidad que poseen los LMS, de llegar a todos los espacios en los cuales exista conexión a internet. De esta forma, se garantiza el acceso a todos los recursos dentro del entorno a quienes, sin importar la distancia, puedan acceder a él.

Según Clarenc, "La ubicuidad en un LMS es la capacidad de una plataforma de hacerle sentir al usuario omnipresente: le transmite la seguridad de que en ella encontrará todo lo que necesita" (Clarenc, 2012, p. 78). De esta forma, las características de los LMS que antes se mencionan dependen de manera directa de esta.

\section{Persuabilidad}

Tal como explica Clarenc (2012 y 2013), la Persuabilidad es una palabra compuesta por dos términos (persuasión y usabilidad) e implica la integración y articulación de cuatro características (Funcionalidad, Usabilidad, Ubicuidad e Interactividad). Edmodo posee una fisionomía semejante a una de las redes sociales más populares: Facebook lo cual permite que por asociación el alumnos se mueva en ella como en un lugar ya común a su memoria. La enumeración de características podría llevarnos más líneas de las permitidas con lo cual, con las seleccionadas se han saturado los elementos más importantes. 
En el caso de edmodo, todas ellas no sólo se cumplen a cabalidad sino que se materializan de manera sensible en los aspectos relacionados con la denominada gestión administrativa de recursos, comunicación y herramientas relacionadas. edmodo, suministra un acceso sencillo al estudiante, así como también al profesor que utiliza la plataforma. Colocando sus datos, (no es necesario que tenga correo electrónico) el alumno se registra y crea su perfil. Posee mecanismos de monitorización que permiten al profesor llevar control del proceso del estudiante, post que coloca, tareas que entrega, rendimiento en las evaluaciones, etc.

A partir del registro del alumno en la plataforma, sus datos quedan registrados en el Libro de calificaciones, un formato en el que su desempeño queda registrado y que además puede ser exportable a otros formatos, como Excel por ejemplo.

En relación al acceso a los recursos y materiales, localizados en carpetas, permiten al estudiante descargarlos de manera sencilla, así como también le dan la posibilidad de subir aportes desde su perfil a la página de inicio que comparte con sus compañeros y profesor. Del mismo modo, la alianza de edmodo con Microsoft y Google le permiten al estudiante almacenar sus trabajos en su drive y al profesor acceder a ellos y colocar los comentarios marginales pertinentes.

Para la comunicación, edmodo ofrece la ventaja de hacerle llegar un correo electrónico al usuario indicándole que se ha hecho una publicación que le compete, le avisa de tareas, así como de las calificaciones obtenidas en las mismas. También ofrece la posibilidad de enviar mensajes colectivos -a grupos completos- o mensajes individuales, a estudiantes o colegas. La pantalla de inicio del curso funciona como un muro en el que se pueden postear informaciones relevantes, así como darle prioridad a algún elemento fijando el mensaje de manera permanente al inicio de la pantalla.

En relación a los datos edmodo permite el almacenamiento de las calificaciones en un libro virtual, en el que puede observarse su desempeño de manera transversal. Ese libro de calificaciones es exportable a diferentes formatos como Word o Excel. En el registro de las evaluaciones el profesor puede realizar un análisis del desempeño de los estudiantes en preguntas específicas de un examen, estableciendo en cuál de las competencias esperadas se observó debilidad.

De la Torre define la Web 2.0 como:

Una forma de entender Internet que, con la ayuda de nuevas herramientas y tecnologías de corte informático, promueve que la organización y el flujo de información dependan del comportamiento de las personas que accedan a ella, permitiéndose no sólo un acceso mucho más fácil y centralizado a los contenidos, sino su propia participación tanto en la clasificación de los mismos como en su propia construcción, mediante herramientas cada vez más fáciles e intuitivas de usar. (De la Torre, 2006, p. 55).

Edmodo desde su distintiva fisionomía permite que los estudiantes accedan a ella de manera casi natural, que creen su perfil, suban su fotografía, accedan a los 
diferentes cursos, adjunten documentación, inserten hipervínculos porque su intuición les dice el camino, en línea con las ventajas previstas en su día por Fombona (2013 y 2016) y según el principio de aprendizaje colaborativo invocado por Pineda Martínez y Castañeda Zumeta (2013), o por Zamora Sánchez et al. (2018).

Edmodo se nos antoja una red social educativa, una comunidad virtual en la que el objetivo fundamental es el aprendizaje de sus miembros. Alonso García, S., Morte Toboso, E. \& Almansa Nuñez, S. (2015), señalan como elementos característicos de una red social con fines educativos los siguientes:

- Aumento del sentimiento de comunidad educativa para alumnos y profesores debido al efecto de cercanía que producen las redes sociales (en la línea de lo sugerido por Marta-Lazo et al., 2018).

- Permiten centralizar en un único sitio todas las actividades docentes, profesores y alumnos de un centro educativo.

- Favorecen la comunicación entre los alumnos, especialmente cuando se forman grupos de trabajo incrementando así las habilidades sociales.

- Acerca la figura del docente a los estudiantes. El profesor ya no resulta tan inaccesible y su localización puede llevarse a cabo fácilmente a través de la red en lugar de otros medios, facilitando el cambio de rol del docente apuntado por Martín-Gómez et al., (2011).

- El aprendizaje resulta más satisfactorio para los estudiantes, incrementándose su grado de motivación ante la posibilidad de ser orientados por otros alumnos o de convertirse en instructores de sus propios compañeros.

- Las herramientas que proporcionan las redes sociales permiten al profesor hacer un seguimiento exhaustivo de su clase, de modo que se convierte en testigo del proceso de trabajo y aprendizaje de sus alumnos.

- Disponen de una amplia variedad de recursos educativos abiertos para estudiantes en formato digital. De esta manera, evitamos el consumo de papel y contribuimos a la reducción de costos en los centros educativos.

- Contribuyen a la formación integral de la persona, ya que se ponen en juego una serie de competencias y habilidades relacionadas con la colaboración, el espíritu crítico y la expresión creativa.

Efectivamente, edmodo suministra a través de su plataforma los mecanismos para ir progresivamente desarrollando ese entramado lúdico en el que se conjugan aquellos elementos de su entorno cotidiano, con oportunidades de aprendizaje que salen de él. La mencionada herramienta permite intervenir de manera positiva la realidad venezolana que se describirá a continuación.

\section{VENEZUELA, DONDE SOBREVIVIR ES LA NORMA}

El siglo XXI, muy a desdén de lo que puedan afirmar estadísticas maquilladas sorprende a la patria de Bolívar en una muy precaria situación. La mencionada precariedad se riega generosa y sin reservas en todos los órdenes de la vida nacional. Economía, Salud y quien más nos ocupa: La Educación, atraviesa uno de los más oscuros momentos de su historia. Con una situación país que ha obligado a muchos al autoexilio el capital humano destinado a formar a otros es cada vez 
menos experto, políticas educativas que lejos de potenciar la excelencia, lo que han hecho es estandarizar la mediocridad y una situación económica de absoluta precariedad dibujan un escenario para nada alentador.

Bravo (2015) realiza un crudo diagnóstico de la situación educativa en Venezuela y los principales signos del comportamiento del Sistema Educativo Escolar, realizado en perspectiva constitucional y de cara a las necesidades de fortalecimiento de la educación inclusivamente democrática.

Sus resultados indican que sus principales indicadores, construidos con la información más oficial disponible, marchan a contracorriente de lo establecido en el Artículo 103 de la Constitución del 99. Uno de los signos que describe de manera prolija es el nivel de exclusión que existe en los grupos escolares.

Asiste actualmente a algún centro de educación/Contactados (Por edades escolares valores absolutos)

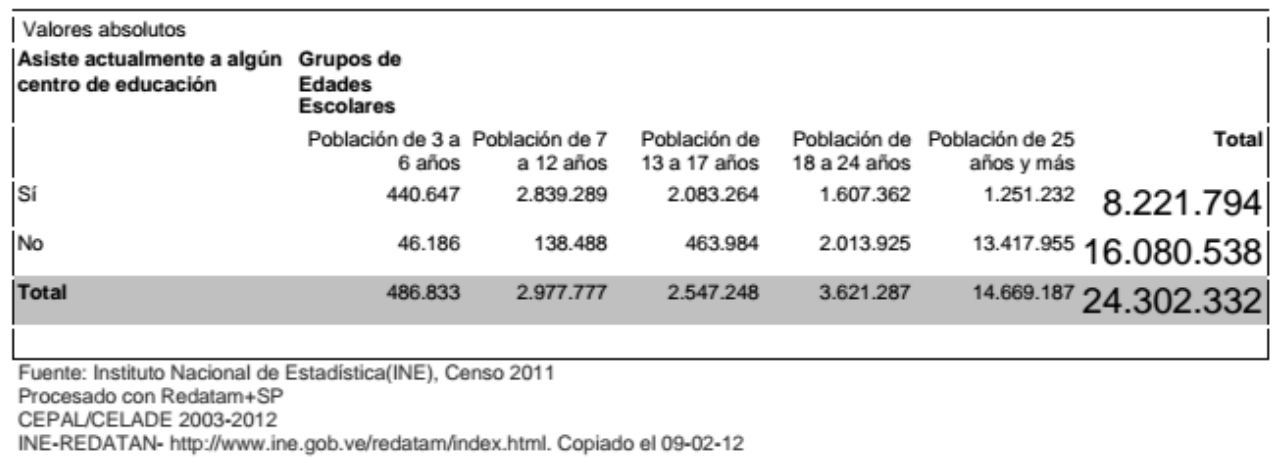

Figura 1: Asistencia a centros de Educación.

Fuente: Instituto Nacional de Estadística (INE), Censo 2011.

Hay exclusión educativa y mucha, afirma el autor, según lo informa el Censo 2011 en términos absolutos respecto a los y las venezolanas que deberían incluidos en algún tipo de actividad educativa. Adicional a esta alarmante realidad debe tomarse en cuenta, está el hecho de la desafiante situación que los hogares venezolanos deben enfrentar a diario para no ser otro punto en esa estadística, con lo que ese porcentaje que logra acceder a la educación pública o privada tiene muchos otros problemas con los que lidiar.

El Diario El Nacional con fecha 15 de Abril de 2016 apuntaba en sus páginas que ese año Venezuela continuaría en recesión económica, se estima que caerá $8 \%$, y 4,5\% en 2017 tras contraerse 5,7\% en 2015. Según los pronósticos del Fondo Monetario Internacional para Latinoamérica, en los cuales ajustó a la baja las predicciones que había emitido en enero. El FMI informó que en la nación caribeña la caída de los precios petroleros profundiza los desequilibrios macroeconómicos preexistentes, por lo cual la inflación será cercana a $500 \%$ en 2016 y a $1.600 \%$ en 2017.

Como complemento a esta difícil circunstancia está el costo de la Canasta Alimentaria Familiar. La Canasta Alimentaria Familiar sólo hace referencia a los 
productos de alimentación, sin tomar en cuenta los productos de higiene personal. Durante el mes de noviembre de 2016 se elevó a 544.990,78 bolívares, este aumento fue de $18,4 \%$ con respecto al mes anterior, equivalente a $84.609,23$ bolívares, es decir, que en la actualidad se necesitan, 20,1 salarios mínimos (27.092,10 bolívares) para adquirir la canasta alimentaria para cinco miembros.

En este sentido, el promedio de las familias en Venezuela tiene diariamente que escoger entre la alimentación de sus miembros y el sortear con cierta dignidad el resto de sus necesidades. La educación y todo lo que ella implica: uniformes, libros, materiales de trabajo, pasan de manera estrepitosa a los últimos lugares en las prioridades.

Como último punto, para no ahondar en otros más dramáticos, como inseguridad y crisis de salud, están las manifestaciones que se presentan en Venezuela, pero especialmente en Caracas, lugar de nuestro estudio. Las manifestaciones -pacíficas o concierto grado de violencia- llegaron a ser en el año 2014, más de 120, de tal manera que un estudiante puede disponerse a ir a su centro educativo, pero alguna de estas contingencias puede impedírselo.

\section{BENEFICIOS DE LA PLATAFORMA EN EL CONTEXTO VENEZOLANO}

En la muy breve descripción en líneas previas, señalábamos tres elementos fundamentales:

- Inclusión en el sistema educativo.

- Limitaciones en el presupuesto familiar.

- Contingencias en el entorno del centro educativo.

En Venezuela, el acceso a literatura especializada es cada vez más complicado y costoso. Del mismo modo, costear materiales educativos es una empresa que coloca en jaque a más de un presupuesto familiar.

La plataforma edmodo tiene un espacio que funciona como biblioteca, tanto personal como del grupo al que el estudiante pertenece. El grupo en el que es estudiante se registra posee un repositorio de materiales denominado: CARPETAS

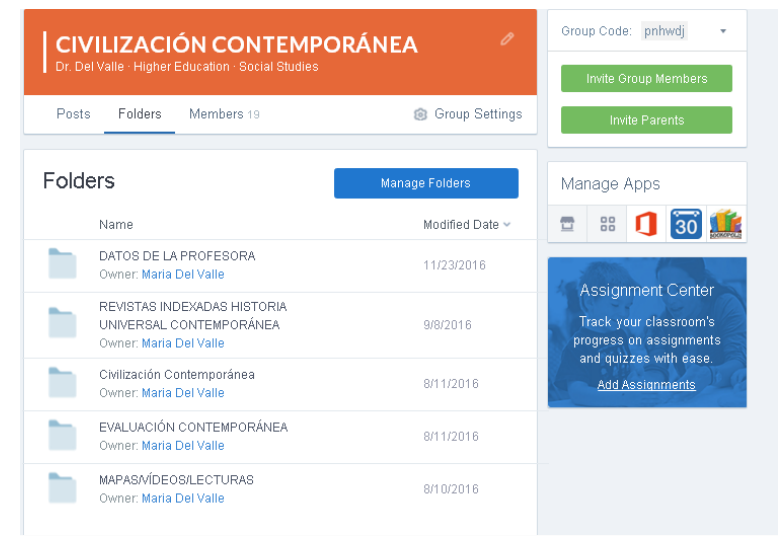

Figura 2: sección de carpetas plataforma de edmodo.

Fuente: elaboración propia. 
En ellas el profesor puede colocar todos los materiales digitales organizados utilizando el criterio que desee. Por temas, por unidades, por tipo de recurso, etc. Igualmente en la pestaña denominada: Bibioteca/Library, el estudiante podrá ir almacenando no solo los materiales que sus maestros le hagan llegar sino también aquellos que él vaya reuniendo según sus intereses.

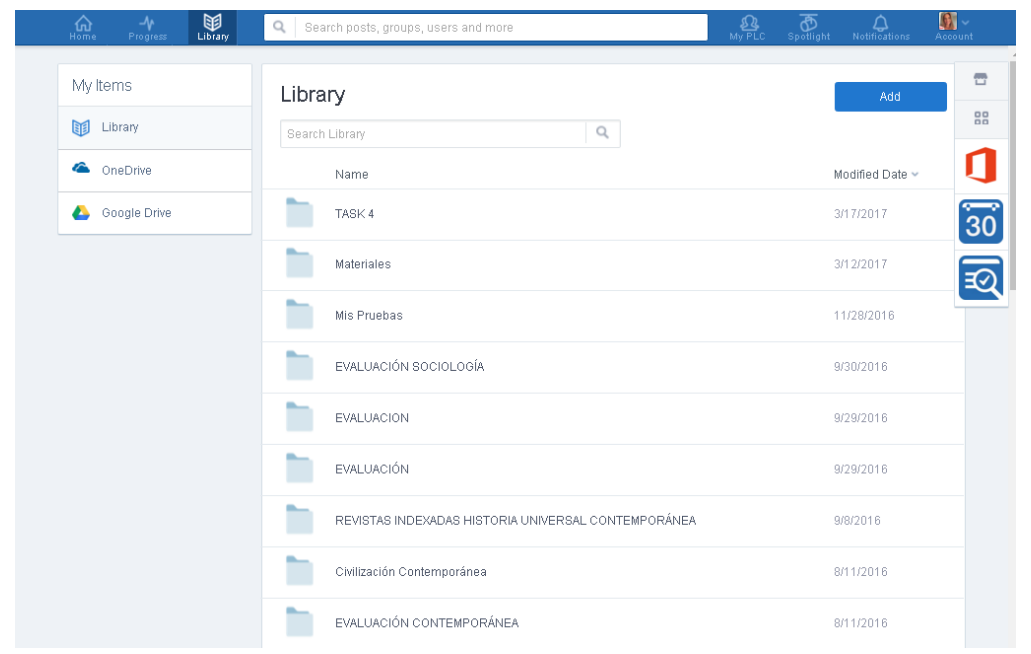

Figura 3: biblioteca plataforma de edmodo.

Fuente: elaboración propia.

Edmodo posee la ventaja de poder hacer vínculo directo con la información almacenada en el Drive de Gmail o en la nube de Microsoft, de esta manera el profesor puede añadir comentarios dentro del mismo documento y que el estudiante lo reciba en línea. Esto no solamente facilita el trabajo sino que implica una reducción importante en los costos de los padres y del estudiante.

Adicionalmente, edmodo posee un repositorio de recursos denominado: Spotlight en el que tanto el estudiante como el alumno encontrarán recursos educativos de última generación para el abordaje de contenidos diversos.

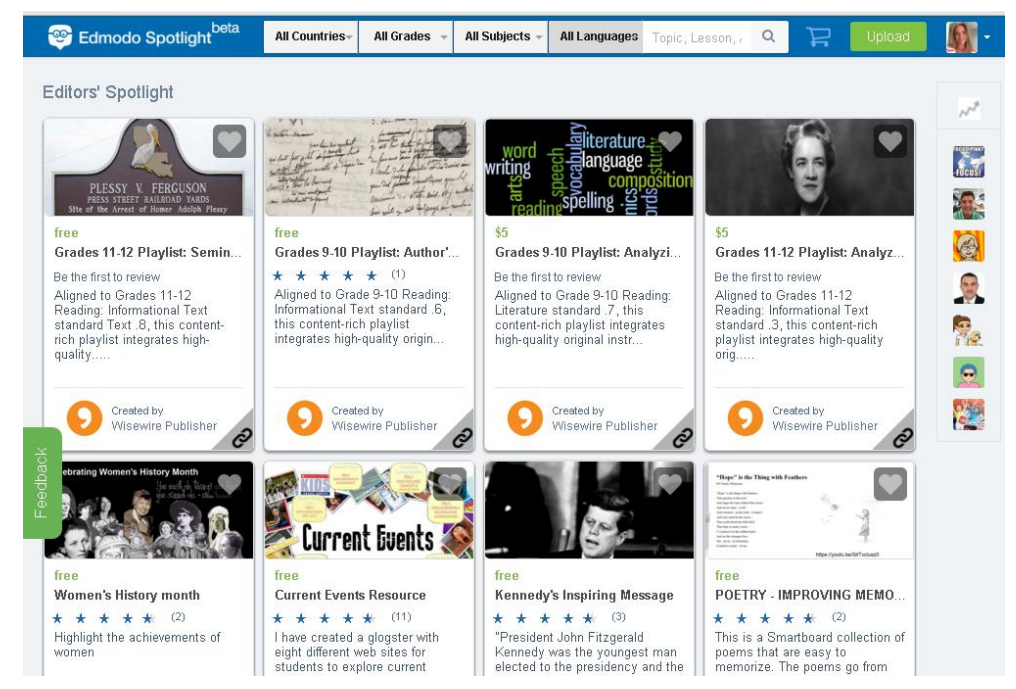

Figura 4: sección de Spotlight plataforma de edmodo.

Fuente: elaboración propia. 
Entre las ventajas que potencian la inclusión en la educación, está la posibilidad que da edmodo para que el estudiante consigne sus trabajos de manera digital sin tener que imprimirlos. Las asignaciones están diseñadas para que el alumno pueda adjuntar sus tareas sin limitarse en el uso de recursos como imágenes, fotografías, gráficos, caricaturas, vínculos a vídeos etc.

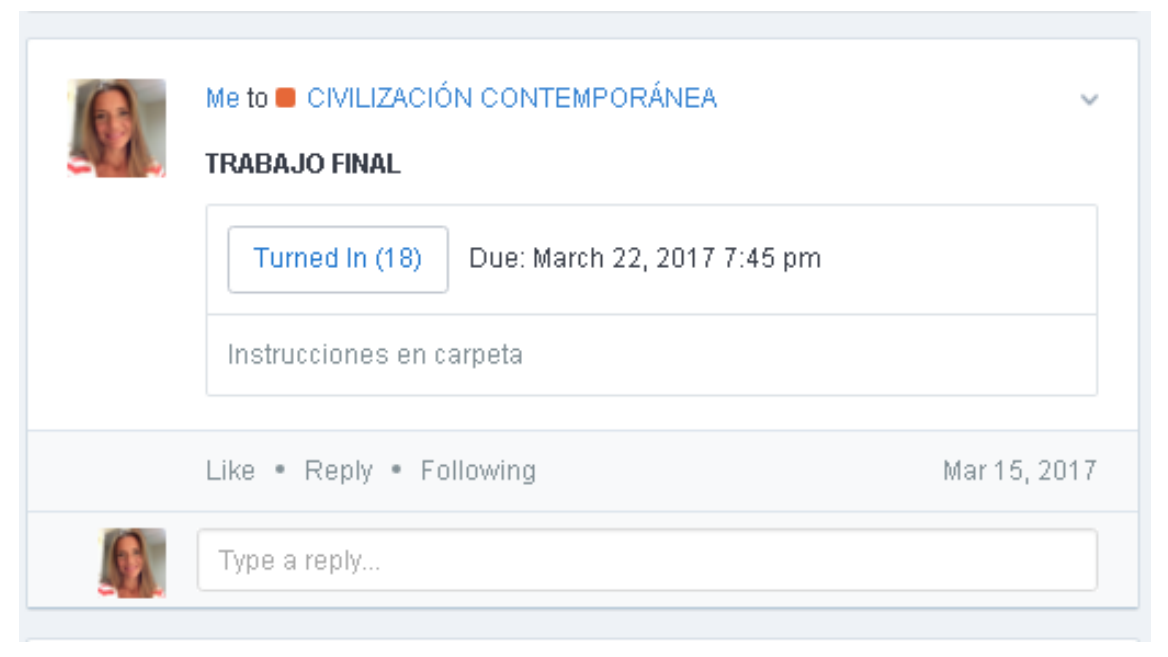

Figura 5: asignaciones plataforma de edmodo.

Fuente: elaboración propia.

Finalmente, la plataforma edmodo se posiciona como un espacio de convergencia comunicacional en el que tanto alumnos como profesores pueden postear notas en el momento que lo consideren oportuno. Pueden enviar mensajes al grupo en general y mensaje a su profesor en particular, lo cual permite hacer del conocimiento de todos cualquier contingencia que imposibilite llegar al aula de clases.

\section{CONCLUSIONES Y RECOMENDACIONES}

Todas las bondades que hemos descrito son sin duda alentadoras, pero como toda herramienta a utilizar tiene sus demandas. Para poder hacer un uso adecuado de la plataforma, así como para generar los materiales que en ella se compartirán, tanto estudiantes como profesores deben recibir capacitación.

Para el logro de este propósito nos hemos estado formando en el manejo de la plataforma y en las complejidades que reviste la generación de materiales para entornos educativos en la web. Estamos convencidos de que herramientas como edmodo, usadas por maestros capacitados, harán bisagra perfecta en los procesos de inclusión que se emprendan desde las políticas públicas o partir de iniciativas generadas por organizaciones no gubernamentales internacionales.

\section{REFERENCIAS}

Alises Camacho, M. E. (2017). Potencial pedagógico del Mobile Learning en el aula de música secundaria. Revista de comunicación de la SEECI, (43), 29-51. Recuperado de http://www.seeci.net/revista/index.php/seeci/article/view/459 
Alonso García, S.; Morte Toboso, E., y Almansa Núñez, S. (2015). Redes sociales aplicadas a la educación: EDMODO. EDMETIC, Revista de Educación Mediática y TIC, 4(2), 88-111.

Beyoda, A. (2007). ¿Qué es interactividad? [Mensaje en un blog]. BLOGS ENAP Universidad Autónoma de México. Recuperado de http://blogs.enap.unam.mx/asignatura/francisco alarcon/wpcontent/uploads/2011/06/interactividad.pdf

Bravo, L. (2015). La escolaridad y la Inversión Educativa en Venezuela al 2015. Recuperado de https://es.scribd.com/document/263148884/Escolaridad-einversion-educativa-en-Venezuela-2015

Clarenc, C. (2012). Videoconferencia: e-Learning-ubicuo - Concepción de ubicuidad en el e-Learning. [Archivo de video]. Recuperado de http://vimeo.com/38286913

Clarenc, C. (2013). Instrumento de evaluación y selección de sistemas de gestión de aprendizaje y otros materiales digitales: Medición y ponderación de LMS y CLMS, recursos educativos digitales y herramientas o sitios de la WEB 3.0. En: Congreso Virtual Mundial de e-Learning: Grupo GEIPITE. Recuperado de http://es.scribd.com/doc/175057118/Instrumento-evaluacion-LMSmaterialesdigitales-recursos-web30

De la Torre, A. (2006). Definición de Web 2.0. [Mensaje en un blog]. Bitácora de Aníbal de la Torre. Recuperado de http://adelat.org/conceptos clave en la web 20 y iii

El Nacional (15 de abril de 2016). FMI: Venezuela cerrará en 2017 con 1600\% de inflación. Recuperado de http://www.el-nacional.com/noticias/economia/fmivenezuela-cerrara-2017-con-1600-inflacion 28189

Fombona Cadavieco, J., y Pascual Sevillano, M. A. (2013). Beneficios del m-learning en la Educación Superior. Educatio Siglo XXI, 31(2), 211-234.

Fombona Cadavieco, J., y Roza Martín, P. (2016). Uso de los dispositivos móviles en educación infantil. Edmetic, 5(2), 159-181.

Marta-Lazo, C.; Valero-Errazu, D., y Gabelas Barroso, J. A. (2018). Uso de Twitter en los MOOC: Nuevas formas de interacción juvenil y su influencia en el aprendizaje. Revista Latina de Comunicación Social, (73), 1333-1352.

Martín-Gómez, S.; García-Rojo, E.; Masa-Lorenzo, C., y Villar-Fernández, N. (2011). Los nuevos modelos de aprendizaje basados en tecnologías de información y comunicación en los grados de administración y dirección de empresas y su aplicación en la Universidad CEU San Pablo. Revista de comunicación VIVAT Academia, (117E), 629-642. doi: http://dx.doi.org/10.15178/va.2011.117E.629$\underline{642}$ 
Pineda Martínez, P., y Castañeda Zumeta, A. (2013). Los LMS como herramienta colaborativa en Educación. Un análisis comparativo de las grandes plataformas a nivel mundial. En: Actas V Congreso Internacional Latina de Comunicación Social V CILCS, pp. 1-13. Universidad de la Laguna, España.

Vaquerizo-García, M. B. (2011). Enseñanza-aprendizaje con web 2.0 y 3.0. Revista de comunicación VIVAT Academia, (117E), 116-121. doi: http://dx.doi.org/10.15178/va.2011.117E.116-121

Zamora Sánchez, R.; Mantilla Falcón, M.; Pullas Tapia, P., y Gómez Alvarado, H. F. (2018). Implicación del modelo de aprendizaje basado en proyectos colaborativos en el contexto universitario. Revista de Comunicación de la SEECI, (46), 01-11. doi: https://doi.org/10.15198/seeci.2018.46.01-11

\section{AUTORA:}

\section{María Elena Del Valle Mejías}

Nacida en Caracas, Venezuela, el 10 de marzo de 1971, graduada de Profesora en la Universidad Pedagógica Libertador en el año 1992, con especialización en Historia Económica y Social de Venezuela en la Universidad Santa María, con Maestría en Historia Económica y Social de Venezuela, tesis con mención publicación y suma cum laude, Doctora en Ciencias de la Educación en el Pedagógico de Caracas, con postdoctorado en Semiótica y Pragmática en la Universidad Complutense de Madrid, cuenta con proyectos financiados por la UPEL y con participación de la Universidad Complutense de Madrid, con proyectos financiados por el FONACIT, investigadora certificada de la Universidad Complutense de Madrid, el grupo internacional CONCILIUM y del CELARG, ponente regular en eventos nacionales e internacionales, adscrita a la línea de investigación, Ciencias del lenguaje y con doce años de investigación en el área de análisis del discurso. Profesora Ordinaria de la Universidad Pedagógica Libertador. Investigadora acreditada del CYTED, la AECID, profesora de la UNIMET. Directora del Master en Comunicación de la TECH University. Profesora Invitada de la Universidad de Vigo. Profesora Honorífica de Universidad Rey Juan Carlos. Colaboradora habitual de los Master en la ESERP y EAE. mvillalba@unimet.edu.ve

Orcid ID: https://orcid.org/0000-0002-8759-6171 